\title{
Diagnosing the Rényi Holographic Dark Energy model in a flat Universe
}

\author{
Vipin Chandra Dubey ${ }^{1 *}$ Ambuj Kumar Mishra ${ }^{2 \dagger}$ Umesh Kumar Sharma $^{3 \ddagger}$ \\ 1,2,3 Department of Mathematics, \\ Institute of Applied Sciences and Humanities, GLA University \\ Mathura-281406, Uttar Pradesh, India.
}

\begin{abstract}
In this paper, we have examined the Rényi holographic dark energy (RHDE) model in the framework of an isotropic and spatially homogeneous flat FLRW (Friedmann- Lemaitre-Robertson-Walker) Universe by considering different values of parameter $\delta$, where the infrared cut-off is taken care by the Hubble horizon. We examined the RHDE model through the analysis of the growth rate of perturbations and the statefinder hierarchy. The evolutionary trajectories of the statefinder hierarchy $S_{3}^{1}, S_{3}^{2} S_{4}^{1}, S_{4}^{2}$ versus redshift $z$, shows satisfactory behaviour throughout the Universe evolution. One of the favourable appliance for exploring the dark energy models is the CND (composite null diagnostic) $\left\{S_{3}^{1}-\epsilon\right\}$ and $\left\{S_{4}^{1}-\epsilon\right\}$, where the evolutionary trajectories of the $\left\{S_{3}^{1}-\epsilon\right\}$ and $\left\{S_{4}^{1}-\epsilon\right\}$ pair show remarkable characteristics and the departure from $\Lambda$ CDM could be very much assessed.
\end{abstract}

Keywords: RHDE; statefinder hierarchy; composite null diagnostic(CND).

PACS: 98.80.Es, 95.36.+x, 98.80.Ck

\section{INTRODUCTION}

Presently cosmologists are facing the problem to understand the reason behind the cosmic acceleration 1, 2]. This can be explained by one of the methods known as the concept of dark energy (DE). But the nature of $\mathrm{DE}$ is not known yet. Various experiments like LSST [3], DES [4, WFIRST [5] and DESI [6] will survey the Universe to understand the nature of DE. Adjoint to these surveys, the accelerated expansion of the universe are supported by various observations like CMBR anisotropies, BAO, SNeIa and LSS formation and $\mathrm{WL}$, which is also consistent with the current standard cosmological model $\Lambda \mathrm{CDM}$, where $\Lambda$ is a constant component of cosmological fluid with the equation of state $\operatorname{EoS} \omega=-1$. Evolution of the cosmological constant is characterized as DE. Since we can only notice the impact of DE on the Hubble flow measuring observable components like radiation and matter. Therefore, we can not evaluate DE directly. Since the DE and gravity are associated in the standard model, we can compute the distribution of matter in the universe by $\omega=p(\rho)$, where $(\rho)$ is the matter-energy density and $p$ is the pressure. For simplicity, we take $\omega=p / \rho$.

Currently, discussion is around on the authenticity of

\footnotetext{
*vipin.dubey@gla.ac.in

†ambuj_math@rediffmail.com

¥ sharma.umesh@gla.ac.in
}

$\Lambda \mathrm{CDM}$ model, which resulting disagreement between Plank 7 and other cosmological measurements like strong lensing time delays (HOLiCOW), megamasers, Cefeids (SHOES), tip of the red giant branch (TRGB) and Oxygenrich Miras and surface brightness fluctuations [8]. These disagreements encourages one to examine other options to the concordance model. To imitate $\Lambda$ according to the cosmological observations at the present time, dynamical dark energy models are most interesting approach. Various approaches are extended theories of gravity [9], Bayesian reconstruction of a time-dependent EoS [10, dark energy parametrisations [11 15], $\omega(z)$ reconstructions [16, modify gravity [17, 18, non-parametric reconstructions of $\omega(z)$ [19, 20], quintessence scenarios 21, 22 and dynamical $\omega_{x}$ from $f(R)$ models [23, 24]. These models helps us in understanding the effects of DE. To describe the accelerated expansion of the cosmos motivated by holograpic principle 25-28, M. Li suggested Holographic dark energy (HDE) where IR cutoff was taken care by future event horizon 29. After that Agegrapic dark enrgy (ADE) model was suggested by Cai by taking length measure as the age of the Universe [30. By considering conformal time as time scale, Wei and Cai suggested the New agegraphic dark energy (NADE) model 31. Gao et al. [32] suggested the Ricci dark energy model by replacing future event horizon with ricci scalar curvature. For investigation of the cosmological and gravitational and incidences recently, different entropies [33 36] has been used to find new form of DE models such as Tsallis holographic dark energy (THDE) model 37, Tsallis agegraphic dark energy (TADE) model [38], Rényi holograpic dark energy (RHDE) model 39] and and Sharma-Mittal holograpic dark energy (SMHDE) model [40. Researchers had used these newly proposed dark energy models in different scenarios 4153 .

Therefore, there is an absolute need for the diagnostic 
tools which can discriminate these various form of dark energy models. Keeping this in mind, different diagnostic tools are proposed to discriminate among various DE models. In 54, authors proposed the growth rate of linear perturbations and statefinder hierarchy, as null diagnostics to differentiate among different dark energy models from $\Lambda$ CDM model. The statefinder hierarchy is a geometrical diagnostic which involves higher-order differential coefficients of scale factor $a(t)$, and also model-independent [55. Statefinder hierarchy has been used to discrimination among BMG (Bimetric Massive Gravity) theory, DGP models and MGG (Modified Galileon Gravity) [56]. To differentiate the HDE models and breaking the degeneracy, the statefinder hierarchy has been investigated in 57]. To discriminate among purely kinetic $k$-essence, modified Chaplygin gas, superfluid Chaplygin gas, generalized Chaplygin gas and $\Lambda \mathrm{CDM}$ model and for analysing the deviation from the $\Lambda \mathrm{CDM}$ model, the statefinder hierarchy and growth rate of perturbation has been used [58, 59. The statefinder hierarchy and the growth rate of perturbations are used in $60-72$. Using statefinder hierarchy in the non-flat Universe considering IR cutoff as apparent horizon has been investigated by one of the authors of present work for the THDE models [73].

In this work, we have explored the newly proposed Rényi Holographic Dark Energy (RHDE) model through the diagnostic tools described above in the flat FRW Universe by taking the Hubble horizon as an infrared cutoff, which has not been explored earlier. Also, we have examined the deviation of the RHDE model from $\Lambda$ CDM using these diagnostic tools. The paper is structured as follows; In Sect. II, we in brief visit the Rényi holographic dark energy. Sect. III is dedicated to discussing the flat FRW cosmological model. Section IV is divided into two subsections A and B, for the methods of the statefinder hierarchy diagnostic and growth rate of perturbations analysis. Finally, in the last section, we have given inferences.

\section{RÉNYI HOLOGRAPHIC DARK ENERGY MODEL}

The form of the Bekenstein entropy of a system is $S=$ $\frac{A}{4}$, where $A=4 \pi L^{2}$ and L is the IR cut-off. Another modified form of the Rényi entropy [74] is given as:

$$
S=\frac{1}{\delta} \log \left(\frac{\delta}{4} A+1\right)=S=\frac{1}{\delta} \log \left(\pi \delta L^{2}+1\right),
$$

Rényi HDE density, by considering the assumption $\rho_{d} d V \propto T d S$, takes the following form:

$$
\rho_{D}=\frac{3 c^{2}}{8 \pi L^{2}}\left(\pi \delta L^{2}+1\right)^{-1}
$$

By taking Hubble horizon as an IR cut-off $L=\frac{1}{H}$, we obtained:

$$
\rho_{D}=\frac{3 c^{2} H^{2}}{8 \pi\left(\frac{\pi \delta}{H^{2}}+1\right)}
$$

where $c^{2}$ is a numerical constant as usual.

\section{THE COSMOLOGICAL MODEL}

For the flat FRW Universe, the metric is given as :

$$
d s^{2}=-d t^{2}+a^{2}(t)\left(d r^{2}+r^{2} d \Omega^{2}\right) .
$$

In a flat FRW Universe, the Friedmann first equation, involving DM and RHDE is defined as :

$$
H^{2}=\frac{1}{3}(8 \pi G)\left(\rho_{D}+\rho_{m}\right)
$$

where $\rho_{D}$ and $\rho_{m}$ represent the energy density of RHDE and matter, respectively. The energy density parameter of RHDE and pressureless matter with the help of the fractional energy densities, can be defined as

$$
\Omega_{m}=\frac{8 \pi \rho_{m} G}{3 H^{2}}, \quad \Omega_{D}=\frac{8 \pi \rho_{D} G}{3 H^{2}},
$$

Now Eq. (5) with help of Eq. (6) can be written as:

$$
1=\Omega_{D}+\Omega_{m}
$$

The conservation law for matter and RHDE are given as :

$$
\begin{gathered}
\dot{\rho}_{m}+3 H \rho_{m}=0, \\
\dot{\rho}_{D}+3 H\left(\rho_{D}+p_{D}\right)=0 .
\end{gathered}
$$

in which $\omega_{D}=p_{D} / \rho_{D}$ represents the RHDE EoS parameter. Now, using differential with time of Eq. (5) in Eq. (8), and Eq. (9) combined the result with the Eq. (7), we get

$$
\frac{\dot{H}}{H^{2}}=\frac{3}{2}\left(\frac{\pi \delta \Omega_{D}}{\pi \delta\left(2 \Omega_{D}-1\right)+\left(\Omega_{D}-1\right) H(t)^{2}}-1\right)
$$

By Eq. 10p, the deceleration parameter (DP) $q$ is found as

$$
\begin{array}{r}
q=-1-\frac{\dot{H}}{H^{2}} \\
q=\frac{(1-2 \delta) \Omega_{D}(t)+1}{2\left(1-(2-\delta) \Omega_{D}(t)\right)}
\end{array}
$$


Also, by taking the derivative with respect to time of Eq. (3), we get

$$
\dot{\rho_{D}}=2 \rho_{D} \frac{\dot{H}}{H}\left(\frac{\pi \delta}{\pi \delta+H^{2}}+1\right)
$$

Now by using the Eqs. 12 with Eqs. (9) and 10 , we gets expression for EoS parameter as:

$$
\omega_{D}=-\frac{\pi \delta}{\pi \delta\left(2 \Omega_{D}-1\right)+H^{2}\left(\Omega_{D}-1\right)} .
$$

Also, taking the time differential of the energy density parameter $\Omega_{D}$ with Eqs. (10) and (12), we find

$$
\Omega_{D}^{\prime}=-\frac{3 \pi c^{2} \delta H^{2}\left(\Omega_{D}-1\right)}{\left(\pi \delta+H^{2}\right)\left(\pi \delta\left(2 \Omega_{D}-1\right)+H^{2}\left(\Omega_{D}-1\right)\right)}
$$

where the dot is the derivative while considering time and prime lets us obtain the derivative concerning ln a.

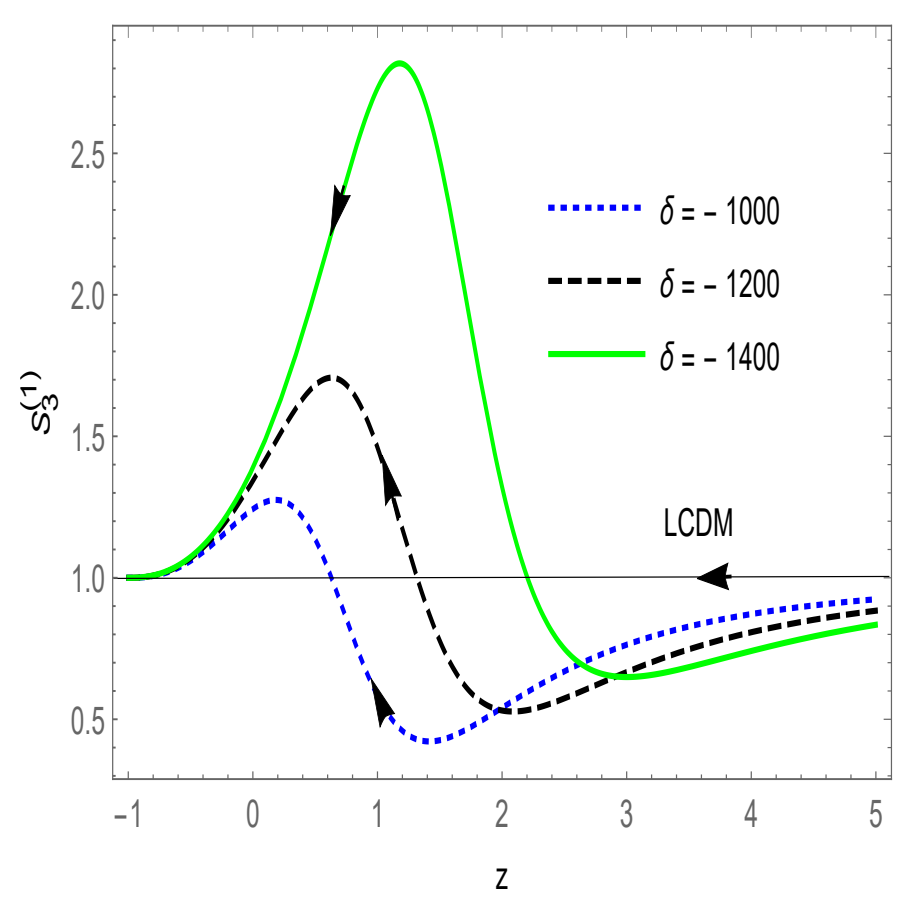

FIG. 1: Graph of $S_{3}^{(1)}$ versus redshift z, for non- interacting RHDE with Hbbble radius as the IR cutoff. Here, $H(z=$ $0)=70, \Omega_{D}(z=0)=0.73$ and different values of $\delta$.

\section{THE METHODS OF DIAGNOSTIC}

In this work, we used two diagnostic tools, statefinder hierarchy and growth rate of perturbations. We shall

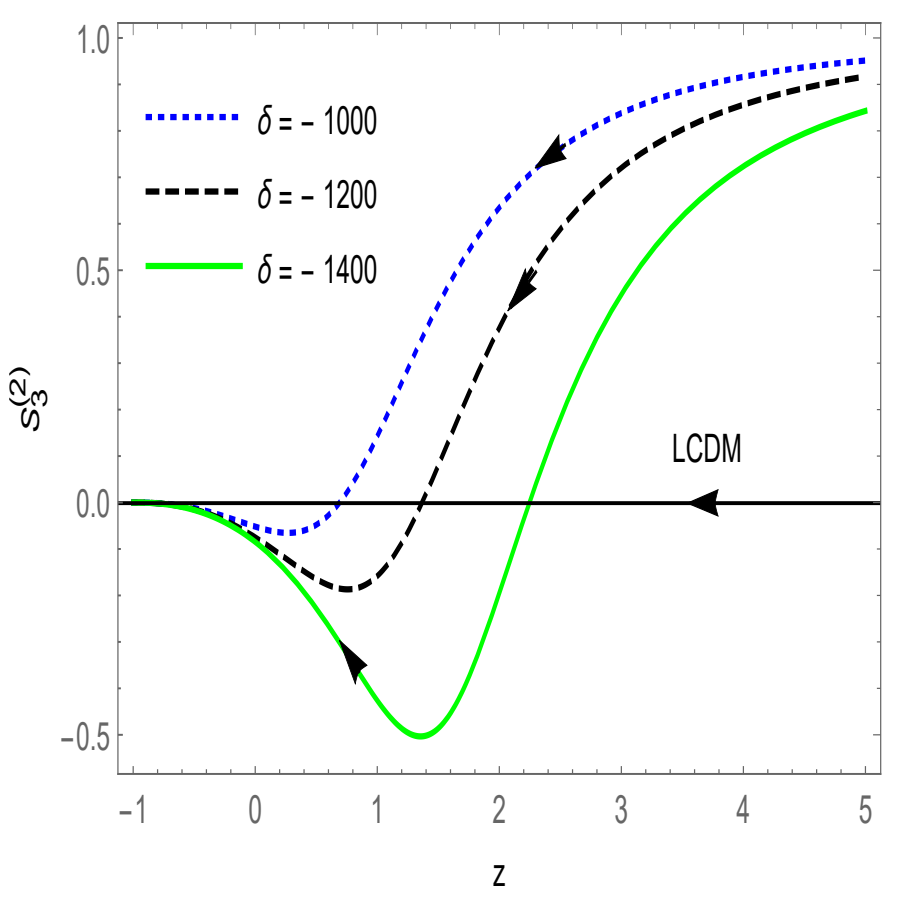

FIG. 2: Graph of $S_{3}^{(2)}$ versus redshift z, for non- interacting RHDE with Hbbble radius as the IR cutoff. Here, $H(z=$ $0)=70, \Omega_{D}(z=0)=0.73$ and different values of $\delta$.

explore the RHDE model to discriminate from $\Lambda$ CDM model with the help of these two diagnostic tools in this section.

\section{A. The Statefinder Hierarchy diagnostic}

Here, statefinder hierarchy diagnostic will be reviewed for the RHDE model will be described. The Taylor expansion of the scale factor $\frac{a(t)}{a_{0}}=\frac{1}{z+1}$, around the present epoch $t_{0}$ is given as:

$$
\frac{a(t)}{a_{0}}=\sum_{n=1}^{\infty} \frac{A_{n}\left(t_{0}\right)}{n !}\left[H_{0}\left(t-t_{0}\right)\right]^{n}
$$

Where $A_{n}=\frac{a^{n}}{a H^{n}}, a^{n}$ is the $n^{t h}$ derivative of the scale factor a verses cosmic time $\mathrm{t}$ and $\mathrm{n} \in \mathrm{N}$. The statefinder hierarchy $S_{n}$ is defined as follows [75]:

$$
S_{2}=A_{2}+\frac{3 \Omega_{m}}{2}, S_{3}=A_{3} \quad \text { and } \quad S_{4}=A_{4}+\frac{9 \Omega_{m}}{2},
$$

Aforementioned gives the diagnostics for the model $(\Lambda \mathrm{CDM})$ with $n \geq 3$, i.e., $S_{n} \mid \Lambda \mathrm{CDM}=1$. Hence by the use of $\Omega_{m}=\frac{2(q+1)}{3}$ the statefinder hierarchy $S_{3}^{(1)}$, $S_{4}^{(1)}$ can be written as:

$$
S_{3}^{(1)}=A_{3}, \quad \text { and } \quad S_{4}^{(1)}=A_{4}+3(q+1)
$$




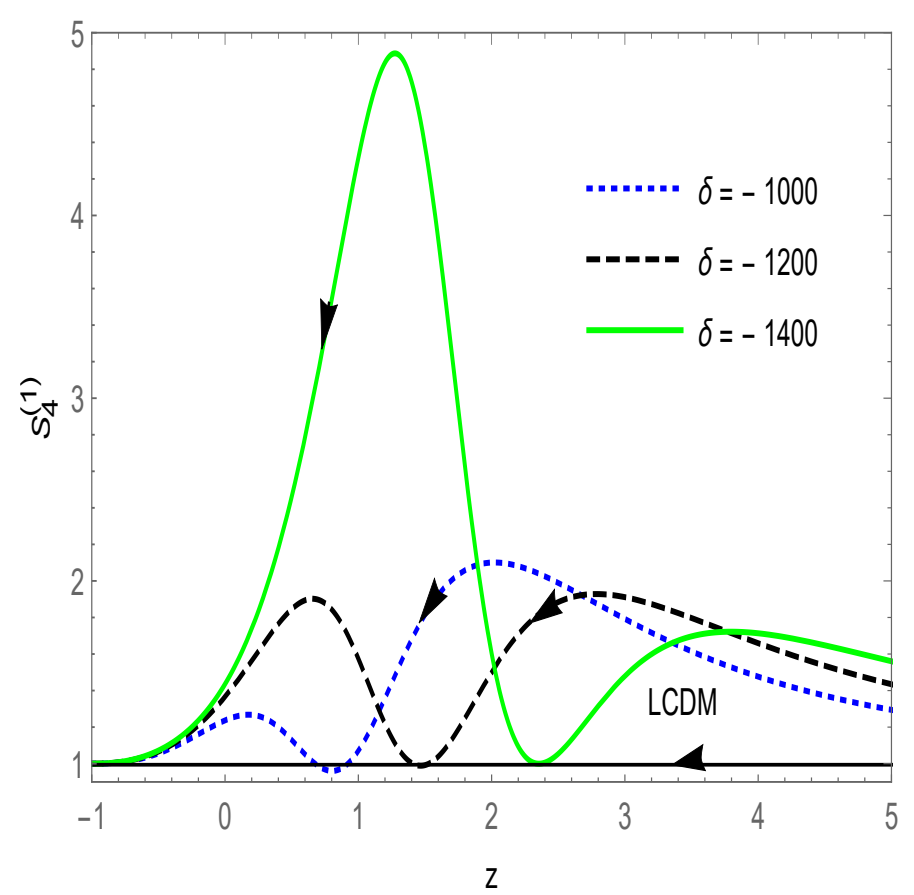

FIG. 3: Graph of $S_{4}^{(1)}$ versus redshift z, for non- interacting RHDE with Hbbble radius as the IR cutoff. Here, $H(z=$ $0)=70, \Omega_{D}(z=0)=0.73$ and different values of $\delta$.

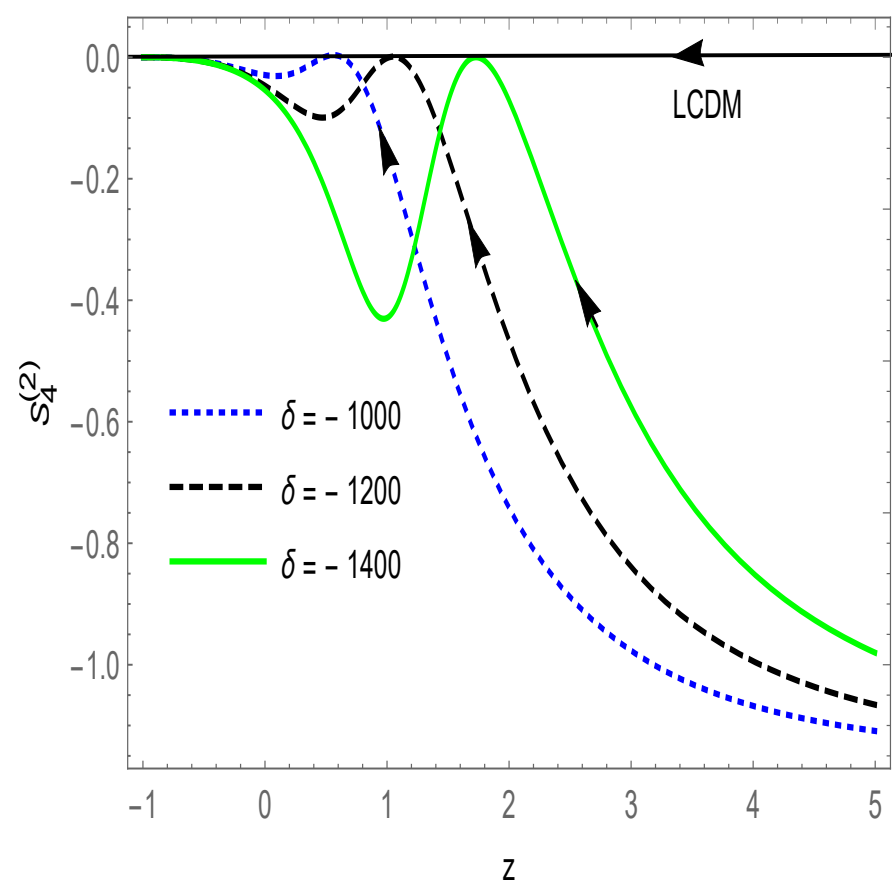

FIG. 4: Graph of $S_{4}^{(2)}$ versus redshift z, for non- interacting RHDE with Hbbble radius as the IR cutoff. Here, $H(z=$ $0)=70, \Omega_{D}(z=0)=0.73$ and different values of $\delta$.
For $\Lambda$ CDM model, $S_{n}^{(1)}=1$. In 72 it gives a path for construction of second Statefinder $S_{3}^{(1)}=S_{3}$ namely

$$
S_{3}^{(2)}=\frac{S_{3}^{(1)}-1}{3\left(q-\frac{1}{2}\right)}
$$

In concordance cosmology $S_{3}^{(1)}=1$ while $S_{3}^{(2)=0}$. Hence, $\left\{S_{3}^{(1)}, S_{3}^{(2)}\right\}=\{1,0\}$ gives a model independent means for forming a distinction between the dark energy models from the cosmological constant [72. Eq. 18] gives the second member of the Statefinder hierarchy

$$
S_{n}^{(2)}=\frac{S_{n}^{(1)}-1}{\alpha\left(q-\frac{1}{2}\right)},
$$

where $\alpha$ is an arbitrary constant. In concordance cosmology $S_{n}^{(2)}=0$ and

$$
\left\{S_{n}^{(1)}, S_{n}^{(2)}\right\}=\{1,0\}
$$

Some of degeneracies in $S_{n}^{(1)}$ can be removed by using the second statefinder $S_{n}^{(2)}$. For the dark energy model, we have

$$
\begin{gathered}
S_{3}^{(1)}=\frac{1}{2}\left(9 \omega_{D}\right)\left(\omega_{D}+1\right) \Omega_{D}+1 \\
S_{3}^{(2)}=\omega_{D}+1 \\
S_{4}^{(1)}=-\frac{1}{4}\left(27 \omega_{D}^{2}\right)\left(\omega_{D}+1\right) \Omega^{2}{ }_{D}^{-} \\
\frac{1}{2}\left(27 \omega_{D}\right)\left(\omega_{D}+1\right)\left(\omega_{D}+\frac{7}{6}\right) \Omega_{D}+1 \\
S_{4}^{(2)}=-\frac{1}{2} \omega_{D}\left(\omega_{D}+1\right) \Omega_{D}-\left(\omega_{D}+1\right)\left(\omega_{D}+\frac{7}{6}\right)
\end{gathered}
$$

where $S_{4}^{(2)}=\frac{S_{4}^{(1)}-1}{9\left(q-\frac{1}{2}\right)}$ and $q-\frac{1}{2}=\frac{1}{2}\left(3 \omega_{D}\right) \Omega_{D}$. As we demonstrate in figures $1,2,3,4$ the Statefinder hierarchy $\left\{S_{n}^{(1)}, S_{n}^{(2)}\right\}$ give us a nice way to differenciating dynamical dark energy models from $\Lambda$ CDM model.

Fig. 1, shows the evolutionary trajectories of $S_{3}^{(1)}(z)$ for the RHDE model by considering different values of $\delta$. The separation of curvilinear shape is more distinct of the RHDE model in the region $0 \leq z \leq 3$ for different values of $\delta$. It is observed that all the curves of $S_{3}^{(1)}(z)$ starts below the $\Lambda$ CDM line $S_{3}^{(1)}=1$ and monotonically increases by crossing the $\Lambda \mathrm{CDM}$ line $S_{3}^{(1)}=1$ form convex vertices in the region $0 \leq z \leq 3$ and then follow the close degeneration together into $\Lambda \mathrm{CDM} S_{3}^{(1)}=1$, at 
low-redshift region. The curves of $S_{3}^{(1)}(z)$ discriminate well from $\Lambda \mathrm{CDM}$ in the high-redshift region but highly degenerate in low-redshift region. This also shows that different values of $\delta$ has quantitative impacts on the $S_{3}^{(1)}(z)$.

Fig. 2, shows the evolutionary trajectories of $S_{3}^{(2)}(z)$ for the RHDE model in the framework of an isotropic and spatially homogeneous flat FRW Universe by considering different values of parameter $\delta$, where the infrared cut-off is taken care by the Hubble horizon. We observe that the evolutionary trajectories of $S_{3}^{(2)}(z)$ are well differentiated from $\Lambda \mathrm{CDM}$ line $S_{3}^{(2)}=0$, at high red-shift region. It starts descending monotonically from the high-redshift region and by crossing the $\Lambda \mathrm{CDM}$ line $S_{3}^{(2)}=0$, forms concave vertices and degenerate closely together with $\Lambda \mathrm{CDM} S_{3}^{(2)}=0$, at low-redshift region. These results shows that different values of $\delta$ has quantitative impacts on the $S_{3}^{(2)}(z)$.

In Fig. 3, we give the graph for $S_{4}^{(1)}$ evolution versus $z$ i.e. redshift for the RHDE model by considering different values of $\delta$. We notice that evolutionary trajectories of $S_{4}^{(1)}$ evolves above the $\Lambda \mathrm{CDM}$ line $S_{4}^{(1)}=1$, at high-redshift region and monotonically increases. Forming first concave vertex by touching the $\Lambda \mathrm{CDM}$ line and again it increases monotonically and forms convex vertices. While the evolutionary trajectories of $S_{4}^{(1)}$ for $\delta=-1200$, crosses the $\Lambda \mathrm{CDM}$ line. Finally these curves degenerate closely together with $\Lambda \mathrm{CDM} S_{4}^{(1)} 1$, at low-redshift region. We notice only quantitative impact on the $S_{3}^{(2)}(z)$ by varying $\delta$.

In Fig. 4, we give the graph for $S_{4}^{(2)}(\mathrm{z})$ evolution versus $z$ i.e. redshift for the RHDE model by considering different values of $\delta$. It is well-differentiated and evolves below the $\Lambda \mathrm{CDM}$ line $S_{4}^{(2)}=0$ at the high red-shift region, and increases monotonically and form convex vertices at $\Lambda \mathrm{CDM}$ line for all values of $\delta$. Then it decreases monotonically, again by making concave vertices finally degenerate closely together with $\Lambda \mathrm{CDM}$ line at the low red-shift region. These evolutionary trajectories shows only quantitative impact on the $S_{4}^{(2)}(z)$ by varying $\delta$.

From figures 1-4, we observe that all the curves superposing at $\Lambda \mathrm{CDM}$ line in the low-redshift region and all the curves separate well in the high-redshift region. So there are two shortcomings in the figures of $S_{3}^{(1)}(z)$ and $S_{4}^{(1)}(z)$. Hence the single diagnostic of geometry is not sufficient. It will be better to combine with the fractional growth parameter, as CND for getting more clear discrimination.

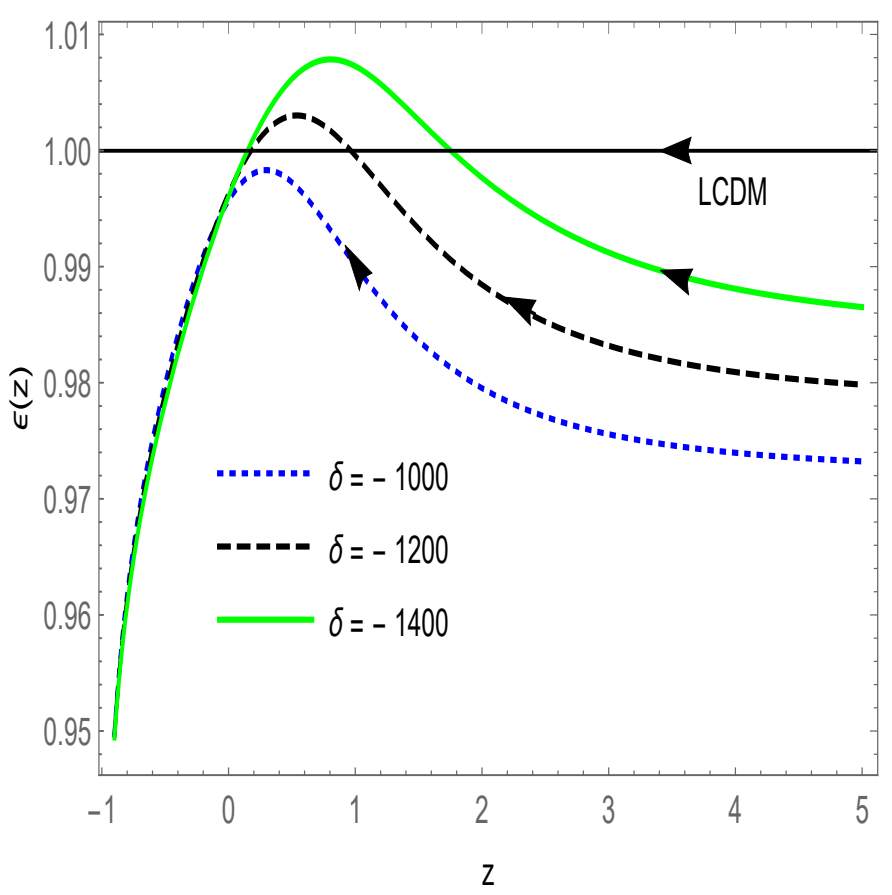

FIG. 5: Graph of $\epsilon(z)$ versus redshift z, for non- interacting RHDE with Hbbble radius as the IR cutoff. Here, $H(z=$ $0)=70, \Omega_{D}(z=0)=0.73$ and different values of $\delta$.

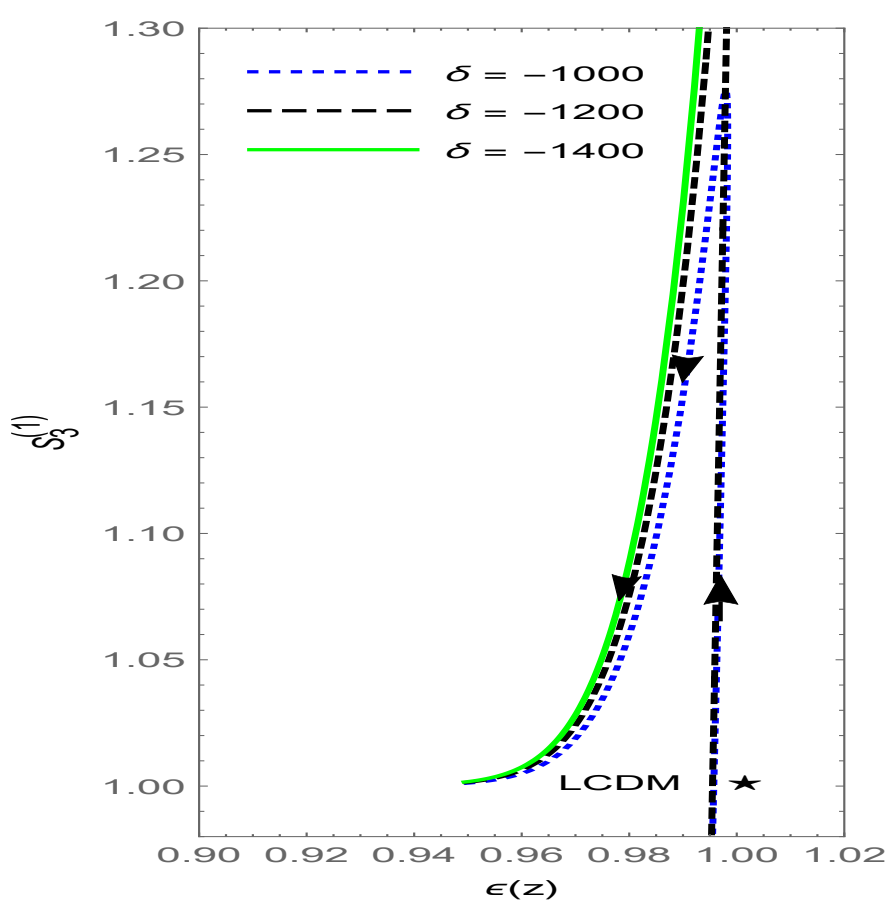

FIG. 6: Graph of $S_{3}^{(1)}$ versus $\epsilon(z)$, for non- interacting RHDE with Hbbble radius as the IR cutoff. Here, $H(z=0)=67$, $\Omega_{m}(z=0)=0.26, R=10000$ and different values of $\delta$ (upper panel) and $H(z=0)=67, \Omega_{m}(z=0) 0.26, \delta=-600$ and different values of $\mathrm{R}$ (below panel). 
TABLE I: The present values of statefinders and fractional growth parameter, $S_{30}^{(1)}, S_{40}^{(1)}$, and $\epsilon_{0}$, and the differences of them, $\triangle S_{30}^{(1)}, \triangle S_{40}^{(1)}$, and $\triangle \epsilon_{0}$

\begin{tabular}{|c|c|c|c|}
\hline$\delta$ & -1000 & -1200 & -1400 \\
\hline$S_{30}^{(1)}$ & 1.24276 & 1.34219 & 1.38857 \\
\hline$S_{40}^{(1)}$ & 1.23685 & 1.36591 & 1.43563 \\
\hline$\epsilon_{0}$ & 0.995679 & 0.996293 & 0.996101 \\
\hline$\triangle S_{30}^{(1)}$ & \multicolumn{3}{|c|}{0.145811} \\
\hline$\triangle S_{40}^{(1)}$ & \multicolumn{3}{|c|}{0.198776} \\
\hline$\triangle \epsilon_{0}$ & \multicolumn{3}{|c|}{0.000421673} \\
\hline
\end{tabular}

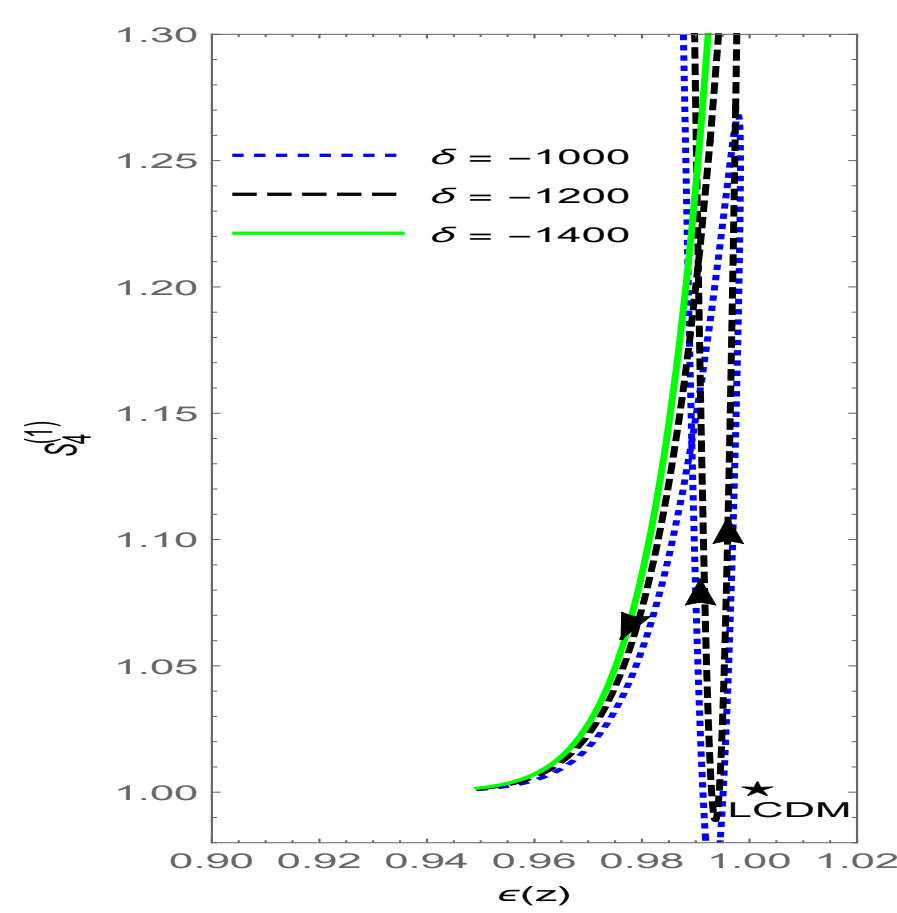

FIG. 7: Graph of $S_{4}^{(1)}$ versus $\epsilon(z)$, for non- interacting RHDE with Hbbble radius as the IR cutoff. Here, $H(z=0)=70$, $\Omega_{D}(z=0)=0.73$ and different values of $\delta$.

\section{B. Growth rate of perturbations}

The fractional growth parameter $\epsilon(z)$ [76, 77] is determined as

$$
\epsilon(z)=\frac{f(z)}{f_{\Lambda \mathrm{CDM}}(z)}
$$

Here $f(z)=\frac{d \log \delta}{d \log a}$ is the growth rate of structure. Here, $\delta=\frac{\delta \rho_{m}}{\rho_{m}}$, with $\delta \rho_{m}$ and $\rho_{m}$ being the the density perturbation and energy density of matter (including CDM and baryons), respectively. If the perturbation is in the linear fashion and without any interaction between DM and DE, then we can say that the equation of perturbation at late times can be:

$$
\ddot{\delta}+2 \dot{\delta} H=4 \pi \delta G \rho_{m}
$$

Here, Newton's gravitational constant is represented by $G$. So, the approx growth rate of linear density perturbation can be reflected by [78:

$$
\begin{gathered}
f(z) \simeq \Omega_{m}(z)^{\gamma} \\
\gamma(z)=\frac{\left(3\left(1-\omega_{D}\right)\left(1-\frac{3 \omega_{D}}{2}\right)\right)\left(1-\Omega_{m}(z)\right)}{125\left(1-\frac{6 \omega_{D}}{5}\right)^{3}}+ \\
\frac{3}{5-\frac{\omega_{D}}{1-\omega_{D}}}
\end{gathered}
$$

where $\Omega_{m}(z)=\frac{\rho_{m}(z)}{3 H(z)^{2} M_{p}^{2}}$, the fractional density of matter, $\Omega$ is constant or varies slowly with time. $\epsilon(z)$ $=1$ and $\gamma \simeq 0.55$ are the values for the $\Lambda \mathrm{CDM}$ model [78, 79]. For other models $\epsilon(z)$ exhibits differences from $\Lambda \mathrm{CDM}$ which would be the possible reason for its use as a diagnostic. By applying the composite null diagnostic $C N D \equiv\left\{S_{n}, \epsilon\right\}$ where $\left\{S_{n}, \epsilon\right\}=\{1,1\}$ for $\Lambda$ CDM, we can make use of both matter perturbational as well as geometrical information of cosmic evolution. While, we can analyze and present only one-side information of cosmic evolution by using one single diagnostic tool.

For the diagnose of diverse theoretical DE models, the evolution of the fractional growth parameter $\epsilon(z)$ is analysed. The evolutionary trajectories of $\epsilon(z)$ versus redshift $z$ for a spatially homogeneous and an isotropic flat FRW Universe of RHDE model by considering different values of $\delta$ are plotted in Fig. 5. It is observed that the evolutionary trajectories of $\epsilon(z)$ evolves below the $\Lambda$ CDM line $\epsilon(z)=1$ and the curves separate well at the high red-shift region. The curves for $\delta=-1200, \delta$ $=-1400$ are monotonically increasing and form convex vertices by crossing the $\Lambda \mathrm{CDM}$ line from past to present. The evolutionary trajectories of $\epsilon(z)$ for $\delta=-1000$, behaves in the same way but it does not cross the $\Lambda$ CDM line. Presently, these curves are degenerated together and decrease monotonically for the low red-shift region.

Fig. 6 shows the evolutionary trajectories of $\left\{S_{3}^{(1)}, \epsilon\right\}$ of RHDE model by considering different values of $\delta$. Where star symbol denotes the $\Lambda$ CDM model $\left\{S_{3}^{(1)}=1, \epsilon=1\right\}$. From figure we observe that, curve evolves near $\Lambda$ CDM model and monotonically increases and forms convex vertices and finally all these curves degenerated together to the line $S_{3}^{(1)}=1$ at low red-shift region.

Fig. 7 is the the evolutionary trajectories of the CND pair $\left\{S_{4}^{(1)}, \epsilon\right\}$ for the RHDE model by considering different values of $\delta$ (upper panel) and $\mathrm{R}$ (below panel). The evolutionary trajectories of $\left\{S_{4}^{(1)}, \epsilon\right\}$ shows similar 
characteristic as the curves of $\left\{S_{3}^{(1)}, \epsilon\right\}$. These results shows that adopting different values of $\delta$ has quantitative impacts and the deviation from $\Lambda \mathrm{CDM}$ can be seen in this figure.

The evolutionary trajectories of the CND pair $\left\{S_{4}^{(1)}, \epsilon\right\}$ for the RHDE model by considering different values of $\delta$ are depicted by Fig. 7. The behaviour of $\left\{S_{4}^{(1)}, \epsilon\right\}$ are similar to the $\left\{S_{3}^{(1)}, \epsilon\right\}$. In the Research work of cosmology, the present values of the parameters keeps important. In this direction we calculated the the present values of parameters $S_{30}^{(1)}, S_{40}^{(1)}$, and $\epsilon_{0}$ of the RHDE model by considering different values of $\delta$ and also the differences of them, for each case $\triangle S_{30}^{(1)}=$ $S_{30}^{(1)}(\max )-S_{30}^{(1)}(\min ), \triangle S_{40}^{(1)}=S_{40}^{(1)}(\max )-S_{40}^{(1)}(\min )$, and $\triangle \epsilon_{0}=\epsilon_{0}(\max )-\epsilon_{0}(\min )$, which is given in Table 1. We observe that $\triangle S_{40}^{(1)}=0.198776>\triangle S_{30}^{(1)}=0.145811$. We can see $\triangle S_{40}^{(1)}>\triangle S_{30}^{(1)}$, which means that the fourth-order derivative of the scale factor in comparison to third-order derivative, intensifies the degeneracy of present values. Therefore $S_{40}^{(1)}$ in comparison to $S_{30}^{(1)}$ gives more variance for different values of $\delta$ among the cosmic evolution of RHDE type of DE. Which helps us to distinguish different theoretical models.

\section{CONCLUSIONS}

The paper uses the Rényi Holographic Dark Energy model in the framework of an isotropic and spatially homogeneous flat FRW Universe by considering different values of RHDE parameter $\delta$, where the infrared cut-off is taken care by the Hubble horizon. This can be summarized as

- In this paper, we examined the deviation of RHDE model from $\Lambda \mathrm{CDM}$ with statefinder hierarchy supplemented by the growth rate of perturbations.

- The statefinder hierarchy $S_{3}^{(1)}, S_{3}^{(2)}, S_{4}^{(1)}$ and $S_{4}^{(2)}$, which contain the third and fourth derivatives of the scale factor, have been plotted versus red-shift z. The evolutionary trajectories of $S_{3}^{(1)}$ evolve below the $\Lambda \mathrm{CDM}$ line while $S_{4}^{(1)}$ evolves from above the $\Lambda$ CDM line. The separation of the curvilinear shape of both parameters is more distinct in the region $0 \leq z \leq 3$ for different values of $\delta$. The evolutionary trajectories of $S_{3}^{(2)}$ evolves above the $\Lambda \mathrm{CDM}$ line and crosses the $\Lambda \mathrm{CDM}$ line but $S_{4}^{(1)}$ evolves below the $\Lambda \mathrm{CDM}$ line and never crosses the $\Lambda \mathrm{CDM}$ line. All parameters degenerated closely together into $\Lambda \mathrm{CDM}$ line, at the low-redshift region.

- We have also examines the growth rate of structure $\epsilon(z)$ by plotting it versus red-shift $\mathrm{z}$ along with the combination of statefinder hierarchy $S_{n}$. The evolutionary trajectories of $\epsilon(z)$ degenerated together and decrease monotonically for low red-shift region. The curves of $\epsilon(z)$ separate well at high red-shift region for different values of $\delta$. The evolutionary trajectories of $\left\{S_{3}^{(1)}, \epsilon\right\}$. and $\left\{S_{4}^{(1)}, \epsilon\right\}$, shows the same deviation from $\Lambda \mathrm{CDM}$ model of RHDE model for all values of $\delta$.

- For alleviating the degeneracy existing in other statefinder parameters for DE models, comparison of the present-value differences of the parameters $S_{30}^{(1)}, S_{40}^{(1)}$ plays an important role. By using CND, we can discriminate RHDE model from $\Lambda$ CDM. Since $\triangle S_{40}^{(1)}>\triangle S_{30}^{(1)}$, hence we can say that the fourth-order hierarchy of statefinder is a better choice than the third-order hierarchy for the RHDE model. Therefore, the above investigation concludes that the higher-order statefinder hierarchy, with the growth rate of perturbations, can differentiate the RHDE model from the $\Lambda$ CDM model and also from itself with different parameter values.

We hope that in future high precision observations, for example, SNAP-type investigation can be equipped for deciding the cosmological parameters exactly and consequently identify the correct cosmological model and closer to understand the properties of the RHDE model.

\section{Acknowledgments}

The authors are thankful for the help and support given by GLA University, Mathura, India, in this research work.
[1] A. G. Riess et al. [Supernova Search Team], "Observational evidence from supernovae for an accelerating universe and a cosmological constant," Astron. J. 116 (1998) 1009 doi:10.1086/300499
[2] S. Perlmutter et al. [Supernova Cosmology Project Collaboration], "Measurements of $\Omega$ and $\Lambda$ from 42 high redshift supernovae," Astrophys. J. 517 (1999) 565 doi:10.1086/307221 
[3] https://www.lsst.org/

[4] https://www.darkenergysurvey.org/

[5] https://wfirst.gsfc.nasa.gov/

[6] http://desi.lbl.gov/

[7] N. Aghanim et al. [Planck Collaboration], arXiv:1807.06209 [astro-ph.CO].

[8] L. Verde, T. Treu and A. G. Riess, arXiv:1907.10625 [astro-ph.CO].

[9] S. Capozziello, R. D'Agostino and O. Luongo, "Extended Gravity Cosmography," Int. J. Mod. Phys. D 28 (2019) no.10, 1930016 doi:10.1142/S0218271819300167

[10] G. B. Zhao et al., "Dynamical dark energy in light of the latest observations," Nat. Astron. 1 (2017) no.9, 627 doi:10.1038/s41550-017-0216-z

[11] I. Sendra and R. Lazkoz, "SN and BAO constraints on (new) polynomial dark energy parametrizations: current results and forecasts," Mon. Not. Roy. Astron. Soc. 422 (2012) 776 doi:10.1111/j.1365-2966.2012.20661.x

[12] G. B. Zhao, D. Bacon, R. Maartens, M. Santos and A. Raccanelli, arXiv:1501.03840 [astro-ph.CO].

[13] C. Escamilla-Rivera, "Status on bidimensional dark energy parameterizations using SNe Ia JLA and BAO datasets," Galaxies 4 (2016) no.3, 8 doi:10.3390/galaxies4030008

[14] M. Rezaei, M. Malekjani, S. Basilakos, A. Mehrabi and D. F. Mota, "Constraints to Dark Energy Using PADE Parameterizations," Astrophys. J. 843 (2017) no.1, 65 doi: $10.3847 / 1538-4357 /$ aa7898

[15] C. Escamilla-Rivera and S. Capozziello, "Unveiling cosmography from the dark energy equation of state," Int. J. Mod. Phys. D 28 (2019) no.12, 1950154 doi:10.1142/S0218271819501542

[16] J. Alberto Vazquez, M. Bridges, M. P. Hobson and A. N. Lasenby, "Reconstruction of the Dark Energy equation of state," JCAP 1209 (2012) 020 doi:10.1088/14757516/2012/09/020

[17] L. G. Jaime, L. Patio and M. Salgado, "Note on the equation of state of geometric dark-energy in f(R) gravity," Phys. Rev. D 89 (2014) no.8, 084010 doi:10.1103/PhysRevD.89.084010

[18] R. Lazkoz, M. Ortiz-Baos and V. Salzano, " $f(R)$ gravity modifications: from the action to the data," Eur. Phys. J. C 78 (2018) no.3, 213 doi:10.1140/epjc/s10052-0185711-6

[19] M. Seikel, C. Clarkson and M. Smith, "Reconstruction of dark energy and expansion dynamics using Gaussian processes," JCAP 1206 (2012) 036 doi:10.1088/1475$7516 / 2012 / 06 / 036$

[20] A. Montiel, R. Lazkoz, I. Sendra, C. Escamilla-Rivera and V. Salzano, "Nonparametric reconstruction of the cosmic expansion with local regression smoothing and simulation extrapolation," Phys. Rev. D 89 (2014) no.4, 043007 doi:10.1103/PhysRevD.89.043007

[21] B. Ratra and P. J. E. Peebles, "Cosmological Consequences of a Rolling Homogeneous Scalar Field," Phys. Rev. D 37 (1988) 3406. doi:10.1103/PhysRevD.37.3406

[22] C. Armendariz-Picon, V. F. Mukhanov and P. J. Steinhardt, "A Dynamical solution to the problem of a small cosmological constant and late time cosmic acceleration," Phys. Rev. Lett. 85 (2000) 4438 doi:10.1103/PhysRevLett.85.4438

[23] S. Capozziello, "Curvature quintessence," Int. J. Mod. Phys. D 11 (2002) 483 doi:10.1142/S0218271802002025

[24] L. G. Jaime, M. Jaber and C. Escamilla-Rivera,
"New parametrized equation of state for dark energy surveys," Phys. Rev. D 98 (2018) no.8, 083530 doi:10.1103/PhysRevD.98.083530

[25] L. Susskind, "The World as a hologram," J. Math. Phys. 36 (1995) 6377 doi:10.1063/1.531249

[26] P. Horava and D. Minic, "Probable values of the cosmological constant in a holographic theory," Phys. Rev. Lett. 85 (2000) 1610 doi:10.1103/PhysRevLett.85.1610

[27] S. D. Thomas, "Holography stabilizes the vacuum energy," Phys. Rev. Lett. 89 (2002) 081301. doi:10.1103/PhysRevLett.89.081301

[28] S. D. H. Hsu, "Entropy bounds and dark energy," Phys. Lett. B 594 (2004) 13 doi:10.1016/j.physletb.2004.05.020

[29] M. Li, "A Model of holographic dark energy," Phys. Lett. B 603 (2004) 1 doi:10.1016/j.physletb.2004.10.014

[30] R. G. Cai, "A Dark Energy Model Characterized by the Age of the Universe," Phys. Lett. B 657 (2007) 228 doi:10.1016/j.physletb.2007.09.061

[31] H. Wei and R. G. Cai, "A New Model of Agegraphic Dark Energy," Phys. Lett. B 660 (2008) 113 doi:10.1016/j.physletb.2007.12.030

[32] C. Gao and F. Wu et al., "A Holographic Dark Energy Model from Ricci Scalar Curvature," Phys. Rev. D 79 (2009) 043511. doi:10.1103/PhysRevD.79.043511

[33] C. Tsallis and L. J. L. Cirto, "Black hole thermodynamical entropy," Eur. Phys. J. C 73 (2013) 2487 doi:10.1140/epjc/s10052-013-2487-6

[34] C. Tsallis, "Possible Generalization of BoltzmannGibbs Statistics," J. Statist. Phys. 52 (1988) 479. doi:10.1007/BF01016429

[35] A.Rényi , in Proceedings of the 4th Berkely Symposium on Mathematics, Statistics and Probability (University California Press, Berkeley, CA, 1961) pp. 547561.

[36] B.D. Sharma and D.P. Mittal, " New non-additive measures of entropy for discrete probability distributions," J. Math. Sci.10 (1975) 28-40; B.D. Sharma, D.P. Mittal, J. Comb. Inf. Syst. Sci. 2 (1977) 122.

[37] M. Tavayef, A. Sheykhi, K. Bamba and H. Moradpour, "Tsallis Holographic Dark Energy," Phys. Lett. B 781 (2018) 195 doi:10.1016/j.physletb.2018.04.001

[38] M. Abdollahi Zadeh, A. Sheykhi and H. Moradpour, "Tsallis Agegraphic Dark Energy Model," Mod. Phys. Lett. A 34 (2019) no.11, 1950086 doi:10.1142/S021773231950086X

[39] H. Moradpour, S. A. Moosavi, I. P. Lobo, J. P. Morais Graa, A. Jawad and I. G. Salako, "Thermodynamic approach to holographic dark energy and the Rnyi entropy," Eur. Phys. J. C 78 (2018) no.10, 829 doi:10.1140/epjc/s10052-018-6309-8

[40] A. Sayahian Jahromi, S. A. Moosavi, H. Moradpour, J. P. Morais Graa, I. P. Lobo, I. G. Salako and A. Jawad, "Generalized entropy formalism and a new holographic dark energy model," Phys. Lett. B $\mathbf{7 8 0}$ (2018) 21 doi:10.1016/j.physletb.2018.02.052

[41] A. Jawad, K. Bamba, M. Younas, S. Qummer and S. Rani, "Tsallis, Rnyi and Sharma-Mittal Holographic Dark Energy Models in Loop Quantum Cosmology," Symmetry 10 (2018) no.11, 635. doi:10.3390/sym10110635

[42] S. Nojiri, S. D. Odintsov and E. N. Saridakis, "Modified cosmology from extended entropy with varying exponent," Eur. Phys. J. C 79 (2019) 242.

[43] Q. Huang, H. Huang, J. Chen, L. Zhang and F. Tu, "Stability analysis of a Tsallis holographic dark energy 
model," Class. Quant. Grav. 36, no. 17, (2019) 175001. S. Ghaffari, H. Moradpour, I. P. Lobo, J. P. Morais Graa and V. B. Bezerra, "Tsallis holographic dark energy in the BransDicke cosmology," Eur. Phys. J. C 78 (2018) no.9, 706 doi:10.1140/epjc/s10052-018-6198-x

[44] E. N. Saridakis, K. Bamba, R. Myrzakulov and F. K. Anagnostopoulos, "Holographic dark energy through Tsallis entropy," JCAP 1812 (2018) 012 doi:10.1088/1475-7516/2018/12/012

[45] V. C. Dubey, S. Srivastava, U. K. Sharma and A. Pradhan, "Tsallis holographic dark energy in Bianchi-I Universe using hybrid expansion law with $k$-essence," Pramana 93 (2019) no.5, 78. doi:10.1007/s12043-019-1843-y

[46] E. Sadri, "Observational constraints on interacting Tsallis holographic dark energy model," Eur. Phys. J. C 79 (2019) no.9, 762 doi:10.1140/epjc/s10052-019-7263-9

[47] E. M. Barboza, Jr., R. d. C. Nunes, E. M. C. Abreu and J. Ananias Neto, "Dark energy models through nonextensive Tsallis statistics," Physica A 436 (2015) 301 doi:10.1016/j.physa.2015.05.002

[48] Golanbari, T., Saaidi, K., Karimi, P. (2020). " Rényi entropy and the holographic dark energy in flat space time". arXiv preprint arXiv:2002.04097.

[49] Sharma, U. K., Dubey, V. C. (2020). "Interacting Rényi holographic dark energy with parametrization on the interaction term". arXiv preprint arXiv:2001.02368

[50] S. Ghaffari, A. H. Ziaie, V. B. Bezerra and H. Moradpour, "Inflation in the Rényi cosmology," Mod. Phys. Lett. A 35 (2019) no.01, 1950341 doi:10.1142/S0217732319503413

[51] V. C. Dubey, et al. "Tsallis holographic dark energy Models in axially symmetric space time." Int. J. Geom. Methods Mod. Phys. 171 (2020) 2050011.

[52] Y. Aditya, S. Mandal, P. K. Sahoo and D. R. K. Reddy, "Observational constraint on interacting Tsallis holographic dark energy in logarithmic BransDicke theory," Eur. Phys. J. C 7912, 1020 (2019).

[53] A. Iqbal and A. Jawad, "Tsallis, Renyi and SharmaMittal holographic dark energy models in DGP brane-world," Phys. Dark Univ. 26 (2019) 100349. doi:10.1016/j.dark.2019.100349

[54] M. Arabsalmani and V. Sahni, "The Statefinder hierarchy: An extended null diagnostic for concordance cosmology," Phys. Rev. D 83 (2011) 043501. doi:10.1103/PhysRevD.83.043501

[55] J. F. Zhang and J. L. Cui et al., "Diagnosing holographic dark energy models with statefinder hierarchy," Eur. Phys. J. C 74 (2014) no.10, 3100. doi:10.1140/epjc/s10052-014-3100-3

[56] R. Myrzakulov and M. Shahalam, "Statefinder hierarchy of bimetric and galileon models for concordance cosmology," JCAP 1310 (2013) 047 doi:10.1088/14757516/2013/10/047

[57] J. F. Zhang, J. L. Cui and X. Zhang, "Diagnosing holographic dark energy models with statefinder hierarchy," Eur. Phys. J. C 74 (2014) no.10, 3100 doi:10.1140/epjc/s10052-014-3100-3

[58] J. L.Cui, et al., " A closer look at interacting dark energy with statefinder hierarchy and growth rate of structure". JCAP 09(2015) 024.

[59] J. Li, R. Yang and B. Chen, "Discriminating dark energy models by using the statefinder hierarchy and the growth rate of matter perturbations," JCAP 1412 (2014) 043 doi:10.1088/1475-7516/2014/12/043
[60] V. Acquaviva and A. Hajian et al., "Next Generation Redshift Surveys and the Origin of Cosmic Acceleration," Phys. Rev. D 78 (2008) 043514. doi:10.1103/PhysRevD.78.043514

[61] V. Acquaviva and E. Gawiser, "How to Falsify the GR+LambdaCDM Model with Galaxy Redshift Surveys," Phys. Rev. D 82 (2010) 082001. doi:10.1103/PhysRevD.82.082001

[62] R. Myrzakulov and M. Shahalam, "Statefinder hierarchy of bimetric and galileon models for concordance cosmology," JCAP 1310 (2013) 047. doi:10.1088/1475$7516 / 2013 / 10 / 047$

[63] J. Li and R. Yang et al., "Discriminating dark energy models by using the statefinder hierarchy and the growth rate of matter perturbations," JCAP 1412 (2014) 043. doi:10.1088/1475-7516/2014/12/043

[64] Y. Hu and M. Li et al., "Impacts of different SNLS3 lightcurve fitters on cosmological consequences of interacting dark energy models," Astron. Astrophys. 592 (2016) A101. doi:10.1051/0004-6361/201526946

[65] A. Mukherjee, N. Paul and H. K. Jassal, "Constraining the dark energy statefinder hierarchy in a kinematic approach," JCAP 1901 (2019) 005 doi:10.1088/14757516/2019/01/005

[66] J. L. Cui, L. Yin, L. F. Wang, Y. H. Li and X. Zhang, "A closer look at interacting dark energy with statefinder hierarchy and growth rate of structure," JCAP 1509 (2015) 024 doi:10.1088/1475-7516/2015/09/024

[67] L. Zhou and S. Wang, "Diagnosing $\Lambda$ HDE model with statefinder hierarchy and fractional growth parameter," Sci. China Phys. Mech. Astron. 59 (2016) no.7, 670411 doi:10.1007/s11433-016-0038-9

[68] Majumdar, A., Chattopadhyay, S., " A study of modified holographic Ricci dark energy in the framework of $\mathrm{f}(\mathrm{T})$ modified gravity and its statefinder hierarchy". Can. J. Phys. 97(5) ( 2018) 477-486.

[69] Zhao, Z., Wang, S., "Diagnosing holographic type dark energy models with the Statefinder hierarchy, composite null diagnostic and $\omega_{D}-\omega_{D}^{\prime}$ pair". China Phys. Mech. Astron. 61(3) (2018) 039811.

[70] Yu, F., et al., "Statefinder hierarchy exploration of the extended Ricci dark energy". Eur. Phys. J. C $\mathbf{7 5}(6)(2015) 274$.

[71] Mukherjee, A., Paul, N., Jassal, H. K., 2019. Constraining the dark energy statefinder hierarchy in a kinematic approach. JCAP 2019(01), 005.

[72] Zhang, N., et al. 2019. Diagnosing the interacting Tsallis Holographic Dark Energy models. arXiv preprint arXiv:1905.04299

[73] Srivastava, V. and Sharma.U. K., "Statefinder hierarchy for Tsallis holographic dark en- ergy." New Astronomy (2020): 101380.

[74] A.Rényi , in Proceedings of the 4th Berkely Symposium on Mathematics, Statistics and Probability (University California Press, Berkeley, CA, 1961) pp. 547561.

[75] V. Sahni and A. Shafieloo et al., "Two new diagnostics of dark energy," Phys. Rev. D 78 (2008) 103502. doi:10.1103/PhysRevD.78.103502

[76] V. Acquaviva and A. Hajian et al., "Next Generation Redshift Surveys and the Origin of Cosmic Acceleration," Phys. Rev. D 78 (2008) 043514. doi:10.1103/PhysRevD.78.043514

[77] V. Acquaviva and E. Gawiser, "How to Falsify 
the GR+LambdaCDM Model with Galaxy Redshift Surveys," Phys. Rev. D 82 (2010) 082001. doi:10.1103/PhysRevD.82.082001

[78] L. M. Wang and P. J. Steinhardt, "Cluster abundance constraints on quintessence models," Astrophys. J. 508
(1998) 483. doi:10.1086/306436

[79] E. V. Linder, "Cosmic growth history and expansion history," Phys. Rev. D 72 (2005) 043529. doi:10.1103/PhysRevD.72.043529 